\title{
ON BOUNDED MATRICES AND KINEMATIC SIMILARITY
}

\author{
BY \\ C. E. LANGENHOP( ${ }^{(1)}$
}

L. Markus [4] has discussed the concept of kinematic similarity of matrices and Lillo [3] has used this idea in the study of almost periodic solutions of differential equations. The concept as considered by Markus and others (see [4] for further references) evidently has its origins in Liapounoff's work [2, pp. 240-243] where the case with which we are concerned in the present paper is referred to as a "reducible system of equations." Markus restricted his considerations to matrix functions of a real variable $t$ which are bounded on a half-line, say $0 \leqq t<\infty$, but almost periodic matrices are bounded on the whole real line. Accordingly we find it more natural, as it would be in the applications Lillo makes, to define $M_{n}$ as the set of all $n$ by $n$ matrices whose entries are complex-valued functions of a real variable $t$ which are continuous and bounded on the whole real line. Let $A(t), B(t)$ $\in M_{n}$. If there exists a $P(t) \in M_{n}$ such that $P^{-1}(t) \in M_{n}$ and $P^{-1}\left(A P-P^{\prime}\right)=B$ (here $P^{\prime}=d P / d t$ ), then we say $A$ is completely kinematically similar to $B$ and we write $A \sim B$. We use the modifier "completely" to distinguish this concept from that considered by Markus, and we shall abbreviate the phrase "completely kinematically similar" to c.k. similar.

Our purpose here is to consider for a matrix $A(t) \in M_{n}$ its c.k. similarity to a constant matrix as a form of characteristic value problem related to linear differential equations involving the matrix $A(t)$. We obtain a necessary and sufficient condition for $A(t) \sim B$, where $B$ is constant, in terms of such problems.

We first make a few observations regarding the differential equations and the solutions involved in c.k. similarity. Suppose $A(t) \in M_{n}$ and $P(t) \in M_{n}$ is such that $P^{-1}(t) \in M_{n}$ and $P^{-1}\left(A P-P^{\prime}\right)=B$ where $B$ is constant. Let $C$ be a nonsingular constant matrix and define $Q(t)=P(t) C$. A trivial calculation shows that $Q^{-1}\left(A Q-Q^{\prime}\right)=C^{-1} B C$ so that $B$ may be put in Jordan canonical form. It is clear then that $A(t) \in M_{n}$ is c.k. similar to a constant matrix if and only if there is a $P(t) \in M_{n}$ and a Jordan matrix $J$ (constant) such that

$$
P^{\prime}=A(t) P-P J
$$

and $P^{-1}(t) \in M_{n}$.

It follows easily that if $P(t)$ is a solution of (1), then $Y(t)=P(t) e^{J t}$ is a solution of

Received by the editors September 9, 1959.

(1) This research was sponsored by the Office of Ordnance Research, U. S. Army. 


$$
Y^{\prime}=A(t) Y,
$$

and if $Y(t)$ is a solution of (2), then $P(t)=Y(t) e^{-J t}$ is a solution of (1). For $Y$ and $P$ related in this way we have $\operatorname{det} P(t)=\operatorname{det} Y(t) \cdot \operatorname{det} e^{-J t}$ and it follows from well known results regarding solutions of linear systems $[1$, p. 28 and p. 76] that

$$
\operatorname{det} P(t)=\operatorname{det} Y(0) \cdot \exp \left[\int_{0}^{t} \operatorname{tr}(A(s)-J) d s\right],
$$

where $\operatorname{det} D=$ determinant of the matrix $D$ and $\operatorname{tr} D=$ trace of $D$. If $P(t)$ is a solution of (1) and $Y(t)=P(t) e^{J t}$, then $P^{-1}(t)$ exists if and only if the columns of $Y$ are linearly independent over the complex numbers since this is true if and only if $\operatorname{det} Y(0) \neq 0\left[1\right.$, p. 69]. Also we observe that if $P^{-1}(t)$ exists, then the columns of $P$ are certainly linearly independent over the complex numbers.

We now state two more observations formally as lemmas. Throughout we assume $A(t) \in M_{n}$ and $J$ constant.

Lemma 1. Let $P(t) \in M_{n}$ be a solution of (1) such that $P^{-1}(t)$ exists. Then $P^{-1}(t) \in M_{n}$ if and only if

$$
\operatorname{Re}\left(\int_{0}^{t} \operatorname{tr}(A(s)-J) d s\right)
$$

is bounded on $-\infty<t<\infty$. (Here $\operatorname{Re}(z)$ denotes the real part of $z$.)

Proof. Clearly $\operatorname{det} P(t) \cdot \operatorname{det} P^{-1}(t)=1$. If $P^{-1}(t)$ is bounded then $\operatorname{det} P(t)$ is bounded away from zero so this with the boundedness of $P(t)$ implies through (3) that (4) holds. Conversely, if (4) holds, then det $P(t)$ is bounded away from zero and this with the boundedness of $P(t)$ implies that $P^{-1}(t)$ is bounded, i.e. $P^{-1}(t) \in M_{n}$.

Lemma 2. Let $P(t) \in M_{n}$ be a solution of (1) such that $P^{-1}(t) \in M_{n}$. Then

$$
\inf |p(t)|>0
$$

for each column vector $p$ of $P$. (Here, if $p^{T}=\left(p_{1 j}, p_{2 j}, \cdots, p_{n j}\right), p^{T}=$ transpose of the column matrix $p$, then $|p(t)|=\left(\sum_{i=1}^{n}\left|p_{i j}(t)\right|^{2}\right)^{1 / 2}$ and the infimum is taken over $-\infty<t<\infty$.)

Proof. Using the Hadamard theorem, $|\operatorname{det} P(t)|^{2} \leqq \prod_{j=1}^{n}\left|p_{j}(t)\right|^{2}$ where $p_{j}(t), j=1,2, \cdots, n$ are the columns of $P$, we see that since the $p_{j}(t)$ are bounded $\left(P \in M_{n}\right)$, if inf $\left|p_{j}(t)\right|=0$ for some $j=1,2, \cdots, n$, then $\inf |\operatorname{det} P(t)|$ $=0$. For $P^{-1}(t) \in M_{n}$ this is impossible by virtue of (3) and Lemma 1 .

The form of the equation (1) and some of the observations made above inspire the following definitions and theorem. 
Definition 1. If $p_{1}, p_{2}, \cdots, p_{r}, r \geqq 1$, are bounded (on $-\infty<t<\infty$ ) vector functions of $t$ such that for the real number $\lambda$

$$
\begin{aligned}
& p_{1}^{\prime}(t)=(A(t)-\lambda I) p_{1}(t), \\
& p_{0}^{\prime}(t)=(A(t)-\lambda I) p_{c}(t)-p_{s-1}(t), \quad s=2,3, \cdots, r,
\end{aligned}
$$

and if

$$
\inf \left|p_{1}(t)\right|>0
$$

then $p_{1}, p_{2}, \cdots, p_{r}$ will be called a characteristic set for $A(t)$ belonging to the characteristic exponent $\lambda$.

Definition 2. If $p_{s}(t, \lambda, i), s=1,2, \cdots, r_{i}(\lambda), i=1,2, \cdots, m$ are characteristic sets for $A(t)$ belonging to the characteristic exponent $\lambda$, then these sets will be said to be independent if $p_{1}(t, \lambda, i), i=1,2, \cdots, m$ are linearly independent over the complex numbers.

Definition 3. If the characteristic exponent $\lambda$ is such that every bounded vector solution of

$$
p^{\prime}=(A(t)-\lambda I) p,
$$

except the identically zero one, satisfies (5), then $\lambda$ will be said to be proper.

DEFinition 4. If there is a maximum number of vectors in a collection of independent characteristic sets for $A(t)$ belonging to the characteristic exponent $\lambda$, this maximum number will be called the multiplicity of $\lambda$.

THEOREM. For $A(t) \in M_{n}$ to be c.k. similar to a constant matrix it is necessary and sufficient that there be real numbers $\lambda_{1}, \lambda_{2}, \cdots, \lambda_{q}$ which are proper characteristic exponents for $A(t)$ and which have multiplicities $\rho_{1}, \rho_{2}, \cdots, \rho_{q}$, respectively, which are such that $\sum_{j=1}^{q} \rho_{j}=n$ and such that $\operatorname{Re}\left(\int_{0}^{t}[\operatorname{tr} A(s)\right.$ $\left.\left.-\sum_{j=1}^{q} \rho_{j} \lambda_{j}\right] d s\right)$ is bounded.

Proof of sufficiency. For each $\lambda_{j}, j=1,2, \cdots, q$ let $p_{s}\left(t, \lambda_{j}, i\right), s=1,2, \cdots$, $r_{i j}, i=1,2, \cdots, m_{j}$ be the independent characteristic sets such that $\sum_{i=1}^{m_{i}} r_{i j}=\rho_{j}$. We may without loss of generality assume that

$$
r_{1 j} \geqq r_{2 j} \geqq \cdots \geqq r_{m_{j} j}, \quad j=1,2, \cdots, q,
$$

and that

$$
\lambda_{1}>\lambda_{2}>\cdots>\lambda_{q} \text {. }
$$

We now form the matrix $P$ using the $p_{s}\left(t, \lambda_{j}, i\right)$ as columns, $p_{s}\left(t, \lambda_{j}, 1\right)$, $s=1,2, \cdots, r_{1 j}$, in order being successive columns followed by $p_{s}\left(t, \lambda_{j}, 2\right)$, $s=1,2, \cdots, r_{2}$, in order etc., these groups of columns being ordered in $P$ according to the index $j$ of $\lambda_{j}$. Since we have $\sum_{j=1}^{g} \sum_{i=1}^{m_{j}} \gamma_{i j}=\sum_{j=1}^{q} \rho_{j}=n$ vectors to form the columns and these vectors are $n$-dimensional, being solutions of (6) and (7), we thus form an $n$ by $n$ matrix $P$. We define 
$J=\operatorname{diag}\left(J_{11}, J_{21}, \cdots, J_{m_{q} q}\right)$ where $J_{i j}=\lambda_{j} I+E, I$ being an $r_{i j}$ by $r_{i j}$ identity matrix and $E$ the same size matrix with ones just above the main diagonal and zeros elsewhere.

Using equations (6) and (7) for the various characteristic sets with $\lambda=\lambda_{j}, j=1,2, \cdots, q$, we find, with $P(t)$ and $J$ as defined above, that $P(t)$ is a solution of (1). Accordingly we form $Y(t)=P(t) e^{t t}$, which is a solution of (2), and show that the columns of $Y(t)$ are linearly independent over the complex numbers. As remarked earlier this implies that $P^{-1}(t)$ exists. The condition that $\operatorname{Re}\left(\int_{0}^{t}\left[\operatorname{tr} A(s)-\sum_{j=1}^{q} \rho_{j} \lambda_{j}\right] d s\right)$ be bounded then implies by Lemma 1 that $P^{-1}(t) \in M_{n}$ so that finally $A(t) \sim J$.

It remains then to examine the columns of $Y=P e^{J t}$. From the form of $e^{J t}[1$, p. 77] the columns of $Y$ may be written

$$
y_{s}\left(t, \lambda_{j}, i\right)=e^{\lambda_{j} t} \sum_{k=0}^{s-1} \frac{t^{k}}{k !} p_{s-k}\left(t, \lambda_{j}, i\right)
$$

for $s=1,2, \cdots, r_{i j}, i=1,2, \cdots, m_{j}, j=1,2, \cdots, q$. Suppose now that for some complex constants $a(s, i, j)$ we have

$$
\sum_{j=1}^{q} \sum_{i=1}^{m_{j}} \sum_{s=1}^{r_{i j}} a(s, i, j) y_{s}\left(t, \lambda_{j}, i\right) \equiv 0 .
$$

We wish to conclude that then all $a(s, i, j)=0$ so we assume they are not. Hence let $j^{\prime}$ be the smallest value of $j=1,2, \cdots, q$ such that $a(s, i, j) \neq 0$ for some $s$ and $i$; thus $a(s, i, j)=0$ for $j<j^{\prime}$. By (10) we have $r_{1 j^{\prime}} \geqq r_{i j^{\prime}}$ for all $i=1,2, \cdots, m_{j^{\prime}}$ so let $s^{\prime}$ be the largest value among the numbers $1,2, \cdots$, $r_{1 j^{\prime}}$ such that $a\left(s, i, j^{\prime}\right) \neq 0$ for some $i$; that is, $a\left(s^{\prime}, i, j^{\prime}\right) \neq 0$ for some $i$ but $a\left(s, i, j^{\prime}\right)=0$ for $s>s^{\prime}$. Next let $i^{\prime}$ be the largest value of $i$ such that $a\left(s^{\prime}, i, j^{\prime}\right)$ $\neq 0$; that is, $a\left(s^{\prime}, i^{\prime}, j^{\prime}\right) \neq 0$ but $a\left(s^{\prime}, i, j^{\prime}\right)=0$ for $i>i^{\prime}$. Finally for each $i>i^{\prime}$ let $s_{i}^{\prime}$ be the largest value of $s \leqq r_{i j^{\prime}}$ such that $a\left(s, i, j^{\prime}\right) \neq 0$. By the definitions of $s^{\prime}$ and $i^{\prime}$ it is clear that $s_{i}^{\prime}<s^{\prime}$ and also that $s^{\prime} \leqq r_{i^{\prime} j^{\prime}}$. Hence by (10) we have $s^{\prime} \leqq r_{i j^{\prime}}$ for $i \leqq i^{\prime}$. With these observations and the notation $z(t ; s, i, j)$ $=a(s, i, j) y_{s}\left(t, \lambda_{j}, i\right)$ we may write (13) in the form

$$
\left(\sum_{i=1}^{i^{\prime}} \sum_{s=1}^{s^{\prime}}+\sum_{i=i^{\prime}+1}^{m_{j^{\prime}}} \sum_{s=1}^{s_{i}{ }^{\prime}}\right) z\left(t ; s, i, j^{\prime}\right)+\sum_{j=j^{\prime}+1}^{q} \sum_{i=1}^{m_{i}} \sum_{s=1}^{r_{i j}} z(t ; s, i, j) \equiv 0,
$$

all other terms being zero.

Using (12), we now substitute for $y_{s}\left(t, \lambda_{j}, i\right)$ in (14) and divide by $t^{s^{\prime}-1} e^{\lambda_{j} t}$ (for $\left.t \neq 0\right)$. The result may be written

$$
\sum_{i=1}^{i^{\prime}} a\left(s^{\prime}, i, j^{\prime}\right) \frac{1}{\left(s^{\prime}-1\right) !} p_{1}\left(t, \lambda_{j^{\prime}}, i\right)+\sum_{1}(t) \equiv 0
$$

where $\sum_{1}(t)$ contains all the terms arising from (14) except those in the very first sum for which $s=s^{\prime}$ and $k=s-1$ where $k$ is as in (12). Thus the 
terms in $\sum_{1}(t)$ are, except for constant factors, of one of the following types:

$$
\begin{gathered}
\exp \left(\left(\lambda_{j}-\lambda_{j^{\prime}}\right) t\right) t^{k+1-s^{\prime}} p_{s-k}\left(t, \lambda_{j}, i\right) \text { with } j>j^{\prime}, \\
t^{k+1-s^{\prime}} p_{s-k}\left(t, \lambda_{j^{\prime}}, i\right) \text { where } i, k, s \text { satisfy (a), (b) or (c), }
\end{gathered}
$$

(a) $i>i^{\prime}, s \leqq s_{i}^{\prime}, k \leqq s-1$,

(b) $i \leqq i^{\prime}, s<s^{\prime}, k \leqq s-1$,

(c) $i \leqq i^{\prime}, s=s^{\prime}, k<s-1$.

Now the functions $p_{s-k}\left(t, \lambda_{j}, i\right)$ are bounded, so certainly terms of the type (16) tend to zero as $t \rightarrow+\infty$ since by (11) $\lambda_{j^{\prime}}>\lambda_{j}$ for $j>j^{\prime}$. For terms of the type (17) we have $k+1-s^{\prime}<0$ in any case so they also tend to zero as $t \rightarrow+\infty$. Hence $\lim _{t \rightarrow+\infty} \sum_{1}(t)=0$. But from (15) we see that $\sum_{1}(t)$ is a solution of (6) with $\lambda=\lambda_{j^{\prime}}$ and since $\lambda_{j^{\prime}}$ is a proper characteristic exponent for $A(t)$ and since inf $\left|\sum_{1}(t)\right| \ngtr 0$ then by Definition $3 \sum_{1}(t) \equiv 0$. But this is impossible since $a\left(s^{\prime}, i^{\prime}, j^{\prime}\right) \neq 0$ and the $p_{1}\left(t, \lambda_{j^{\prime}}, i\right), i=1,2, \cdots, m_{j^{\prime}}$, are linearly independent by Definition 2 , the characteristic sets belonging to each $\lambda_{j}$ being independent by hypothesis. This contradiction implies $a(s, i, j)=0$ for all $s, i$ and $j$ so the $y_{s}\left(t, \lambda_{j}, i\right)$ are linearly independent as was to be shown and the proof of sufficiency is completed.

Proof of necessity. We assume $A(t)$ is c.k. similar to a constant matrix which, as pointed out earlier, may be taken to be in Jordan canonical form. Hence there is a $P(t) \in M_{n}$ and a Jordan matrix $J$ which satisfy equation (1). In fact, as Markus indicates, $J$ may be taken real, for if $J=\operatorname{diag}\left(J_{1}, J_{2}, \cdots, J_{q}\right)$ where $J_{j}$ has only the characteristic root $\lambda_{j}$ let $\mu_{j}$ be the imaginary part of $\lambda_{j}, j=1,2, \cdots, q$. Then $S=\operatorname{diag}\left(\mu_{1} I, \mu_{2} I, \cdots, \mu_{q} I\right)$ and $e^{i S t}$ commute with $J$ if the scalar blocks $\mu_{j} I$ in $S$ are the same size as the corresponding block $J_{j}$ in $J$. Define $Q=P e^{i S t}$ and we have

$$
Q^{\prime}=A Q-Q(J-i S)
$$

where now $J-i S$ is in Jordan form and has only real characteristic roots. Accordingly we assume that $J$ in (1) is real. If $\lambda_{1}, \lambda_{2}, \cdots, \lambda_{q}$ are the distinct characteristic roots of $J$ we may order them as in (11) and the sizes $r_{i j}$ of the various elementary divisor blocks as in (10) and take $J$ as it was in the proof of sufficiency.

Since $P(t) \in M_{n}$ and $P^{-1}(t) \in M_{n}$ it follows by Lemma 2 that inf $|p(t)|>0$ for every column of $P(t)$. Hence from the form of (1) it is clear that by Definition 1 the columns of $P(t)$ make up a collection of characteristic sets for $A(t)$ belonging to the characteristic exponents $\lambda_{1}, \lambda_{2}, \cdots, \lambda_{q}$. Moreover since $P^{-1}(t)$ exists the columns of $P$ must be linearly independent over the complex numbers so that the characteristic sets for each $\lambda_{j}$ are independent according to Definition 2. Also with $\rho_{j}=\sum_{i=1}^{m_{j}} r_{i j}$ the multiplicity of $\lambda_{j}$ is at least $\rho_{j}$. We must show that the multiplicity of $\lambda_{j}$ is equal to $\rho_{j}, j=1,2, \cdots, q$ and that the $\lambda_{j}$ are proper characteristic exponents. This would complete the 
proof since clearly $\sum_{j=1}^{g} \rho_{j}=n$ and since $P^{-1}(t) \in M_{n}$ we have by Lemma 1 that $\operatorname{Re}\left(\int_{0}^{q}\left[\operatorname{tr} A(s)-\sum_{j=1}^{q} \rho_{j} \lambda_{j}\right] d s\right)$ is bounded.

To show that the characteristic exponents $\lambda_{j}, j=1,2, \cdots, q$ are proper we first consider vectors $\psi(t)$ of the form

$$
\psi(t)=\sum_{i=1}^{m_{j^{\prime}}} c_{i} p_{1}(t, \lambda, i)
$$

where $j^{\prime}$ is one of the numbers $1,2, \cdots, q$ and $\lambda=\lambda_{j^{\prime}}$. We shall show that inf $|\psi(t)|>0$ unless all the $c_{i}$ are zero. If not all the $c_{i}$ are zero we show that $\psi(t)$ may be taken to be a column in a matrix $\Psi(t) \in M_{n}$ such that $\Psi^{-1}(t) \in M_{n}$ and $\Psi^{\prime}(t)=A(t) \Psi(t)-\Psi(t) J$ and it follows from Lemma 2 then that inf $|\psi(t)|$ $>0$. In fact, we define $\Psi(t)=P(t) K$ where $K$ is a nonsingular constant matrix which commutes with $J$ and the resulting $\Psi$ will have the properties just mentioned.

For simplicity of notation let $r_{i}=r_{i j^{\prime}}$ and $m=m_{j^{\prime}}$ and because of the ordering (10) we have $r_{1} \geqq r_{2} \geqq \ldots \geqq r_{m}$. Let $\sigma$ be the largest value of $i$ for which $c_{i} \neq 0$ in (18), i.e. $c_{\sigma} \neq 0$ but $c_{i}=0$ for $i>\sigma$. Then $r_{i} \geqq r_{\sigma}$ for $i \leqq \sigma$. Now we write $J$ in block form as

$$
J=\left(\begin{array}{lll}
J_{\alpha} & 0 & 0 \\
0 & J^{\prime} & 0 \\
0 & 0 & J_{\beta}
\end{array}\right),
$$

where $J_{\alpha}$ and $J_{\beta}$ contain the elementary divisor blocks $J_{i j}$ for which $j<j^{\prime}$ and $j>j^{\prime}$ respectively and $J^{\prime}$ contains those blocks for which $j=j^{\prime}$. Then we have $J^{\prime}$ in block form as

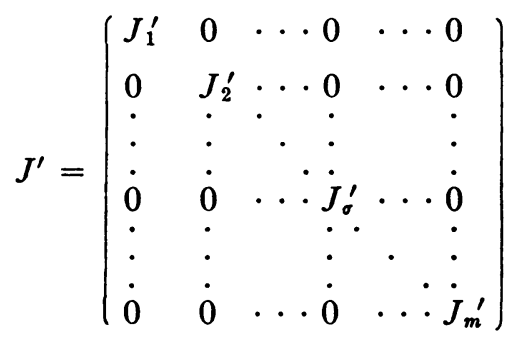

where, again for simplicity in notation, $J_{i}^{\prime}=J_{i j^{\prime}}, i=1,2, \cdots, m$; i.e. $J_{i}^{\prime}=\lambda I+E$ where $I$ and $E$ are as before and are $r_{i}$ by $r_{i}$ in size. For $K$ we take

$$
K=\left(\begin{array}{lll}
I & 0 & 0 \\
0 & K^{\prime} & 0 \\
0 & 0 & I
\end{array}\right),
$$

where the partitioning is as in (19). For $K^{\prime}$ we take 


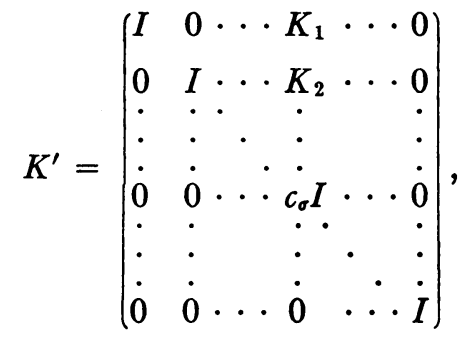

where the partitioning is as in (20). The matrices $K_{i}$ are $r_{i}$ rows by $r_{\sigma}$ columns and we take

$$
K_{i}=\left(\begin{array}{l}
c_{i} I \\
0
\end{array}\right), \quad \text { for } i<\sigma,
$$

where here $I$ is an $r_{\sigma}$ by $r_{\sigma}$ identity matrix. The diagonal blocks in $K^{\prime}$ are all identity blocks except for $c_{\sigma} I$ in the $\sigma$ th place, all blocks above this one are to be of the form (23) and all other blocks are zero blocks.

From the form of $J$ and $K$ in (19) and (21) we need only verify $J^{\prime} K^{\prime}=K^{\prime} J^{\prime}$ in order that $J K=K J$ and from the form of $J^{\prime}$ and $K^{\prime}$ in (20) and (22) we need only verify that $J_{i}^{\prime} K_{i}=K_{i} J_{\sigma}^{\prime}$ for $i<\sigma$ in order that $J^{\prime} K^{\prime}=K^{\prime} J^{\prime}$. But for $i<\sigma, r_{i} \geqq r_{\sigma}$ so we may write

$$
J_{i}^{\prime}=\left(\begin{array}{cc}
\lambda I+E & W \\
0 & \lambda I+E
\end{array}\right),
$$

where $W$ has $r_{\sigma}$ rows and $r_{i}-r_{\sigma}$ columns, and using (23) the relation $J_{i}^{\prime} K_{i}$ $=K_{i} J_{\sigma}^{\prime}$ is easily verified. Thus $J K=K J$. Now from (22) we see that $K^{\prime}$ is upper triangular and we have from (21) det $K=c_{\sigma} r_{\sigma}$. But $c_{\sigma} \neq 0$ so $K$ is nonsingular. Now it is clear from the prescribed form of $K$ that $\psi(t)$ in (18) is one of the columns of $\Psi(t)=P(t) K$. Hence it follows that inf $|\psi(t)|>0$.

With the result just established we can finish the proof that the $\lambda_{j}, j=1,2, \cdots, q$, are proper characteristic exponents for $A(t)$. Again let $\lambda=\lambda_{j^{\prime}}$ where $j^{\prime}$ is one of the numbers $1,2, \cdots, q$ and define $Z(t)=P(t) e^{(J-\lambda I) t}$. Since $P(t)$ satisfies (1) it is readily verified that $Z(t)$ satisfies

$$
Z^{\prime}=(A(t)-\lambda I) Z \text {. }
$$

Moreover since $P(t)$ is nonsingular so also is $Z(t)$ and the columns of $Z(t)$ span the space of all solutions of

$$
p^{\prime}=(A(t)-\lambda I) p .
$$

The columns of $Z(t)$ may be written as

$$
z_{s}\left(t, \lambda_{j}, i\right)=\exp \left(\left(\lambda_{j}-\lambda\right) t\right) \sum_{k=0}^{s-1} \frac{t^{k}}{k !} p_{s-k}\left(t, \lambda_{j}, i\right),
$$


for $s=1,2, \cdots, r_{i j}, i=1,2, \cdots, m_{j}, j=1,2, \cdots, q$. Now let $\phi(t)$ be a bounded solution of (26). It may be written as

$$
\phi(t)=\sum_{j=1}^{q} \sum_{i=1}^{m_{j}} \sum_{s=1}^{r_{i j}} b(s, i, j) z_{s}\left(t, \lambda_{j}, i\right) .
$$

By reasoning similar to that in the sufficiency proof we may now show that all $b(s, i, j)=0$ except possibly those for which $s=1$ and $j=j^{\prime}$. For if we assume $b(s, i, j) \neq 0$ for some $s$ and $i$ for some $j \neq j^{\prime}$ we take $j^{\prime \prime}$ to be the largest value of $j>j^{\prime}$ or the smallest value of $j<j^{\prime}$ such that $b(s, i, j) \neq 0$ for some $s$ and $i$. We then let $s^{\prime \prime}$ be the largest value of $s$ such that $b\left(s, i, j^{\prime \prime}\right) \neq 0$ for some $i$ and let $i^{\prime \prime}$ be the largest value of $i$ such that $b\left(s^{\prime \prime}, i, j^{\prime \prime}\right) \neq 0$. Thus $b\left(s^{\prime \prime}, i^{\prime \prime}, j^{\prime \prime}\right) \neq 0$ but $b\left(s^{\prime \prime}, i, j^{\prime \prime}\right)=0$ for $i>i^{\prime \prime}$ and $b\left(s, i, j^{\prime \prime}\right)=0$ for $s>s^{\prime \prime}$. After substituting from (27) into (28) and dividing the result by $t^{s^{\prime \prime}-1} \exp \left(\left(\lambda_{j^{\prime \prime}}-\lambda\right) t\right)$, we may write the result in the form

$$
\sum_{i=1}^{i^{\prime \prime}} b\left(s^{\prime \prime}, i, j^{\prime \prime}\right) \frac{1}{\left(s^{\prime \prime}-1\right) !} p_{1}\left(t, \lambda_{j^{\prime \prime}}, i\right)=\sum_{2}(t)+t^{1-8^{\prime \prime}} e^{\left(\lambda-\lambda_{j^{\prime \prime}}\right) t} \phi(t),
$$

where $\sum_{2}(t)$ contains all the terms arising from those in (28) except those for which $j=j^{\prime \prime}, i \leqq i^{\prime \prime}, s=s^{\prime \prime}$ and $k=s-1$ where $k$ is as in (27). The limit of the right hand side of (29) is zero as $t \rightarrow+\infty$ or as $t \rightarrow-\infty$ according as $j^{\prime \prime}<j^{\prime}$ or $j^{\prime \prime}>j^{\prime}$ respectively. But the left hand side is a linear combination of the vectors $p_{1}\left(t, \lambda_{j^{\prime \prime}}, i\right)$ for which at least $b\left(s^{\prime \prime}, i^{\prime \prime}, j^{\prime \prime}\right) \neq 0$ and, as shown above, must then be bounded away from zero. This is a contradiction so we conclude that all $b(s, i, j)=0$ in (28) except possibly those for which $j=j^{\prime}$. Hence we must have

$$
\phi(t)=\sum_{i=1}^{m} \sum_{s=1}^{r_{i}} b\left(s, i, j^{\prime}\right) \sum_{k=0}^{s-1} \frac{t^{k}}{k !} p_{s-k}(t, \lambda, i)
$$

where again $m=m_{j^{\prime}}, r_{\imath}=r_{i j^{\prime}}$ and $\lambda=\lambda_{j^{\prime}}$.

By a similar argument we can now conclude that all $b\left(s, i, j^{\prime}\right)=0$ except possibly $b\left(1, i, j^{\prime}\right)$ for some values of $i$. Thus (30) reduces to

$$
\phi(t)=\sum_{i=1}^{m} b\left(1, i, j^{\prime}\right) p_{1}(t, \lambda, i)
$$

and again by nur previous result inf $|\phi(t)|>0$ unless $b\left(1, i, j^{\prime}\right)=0, i=1,2$, $\cdots, m$, i.e. unless $\phi(t) \equiv 0$. Hence we conclude that $\lambda$ is a proper characteristic exponent for $A(t)$ and since $\lambda$ represented any arbitrary one of the $\lambda_{j}$ the fact that all characteristic exponents are proper is established.

It remains to show that the multiplicities of the characteristic numbers must indeed be $\rho_{j}$. If $n_{j}$ is the multiplicity of $\lambda_{j}$, then $n_{j} \geqq \rho_{j}$, as has already been remarked. However, it is clear from the sufficiency proof that given a 
collection of independent characteristic sets belonging to proper characteristic exponents for $A(t)$, their multiplicities $n_{j}$ must satisfy $\sum_{j=1}^{q} n_{j} \leqq n$ since there can be at most $n$ linearly independent vectors defined through such sets by (12). Hence $\sum_{i=1}^{q} \rho_{j} \leqq \sum_{i=1}^{q} n_{j} \leqq n$. But we now clearly have $\sum_{i=1}^{q} \rho_{j}$ $=n$ in case $A(t)$ is c.k. similar to a constant matrix. Thus none of the inequalities $n_{j}>\rho_{j}$ can hold so $n_{j}=\rho_{j}$ for $j=1,2, \cdots, q$. This completes the necessity proof.

REMARKs. The vectors $y_{s}\left(t, \lambda_{j}, i\right)$ defined by (12) are precisely those solutions of $x^{\prime}=A(t) x$ which exhibit the invariants discussed by Markus [4]. To see this we may write

$$
y_{s}\left(t, \lambda_{j}, i\right)=e^{\lambda_{j} t} t^{s-1}\left[\frac{1}{(s-1) !} p_{1}\left(t, \lambda_{j}, i\right)+\sum_{k=0}^{s-2} \frac{t^{k+1-s}}{k !} p_{s-k}\left(t, \lambda_{j}, i\right)\right] .
$$

Since the $p_{s}\left(t, \lambda_{j}, i\right)$ are bounded the terms after the summation sign in the bracketed expression tend to zero as $t \rightarrow \pm \infty$. But inf $\left|p_{1}\left(t, \lambda_{j}, i\right)\right|>0$ so the norm of the bracketed expression is bounded and bounded away from zero for all sufficiently large $t$. Thus for $t \geqq T$ for some $T$ we have

$$
\log \left|y_{s}\left(t, \lambda_{j}, i\right)\right|=\lambda_{j} t+(s-1) \log t+\log |b(t)|
$$

where $b(t)$ is the bracketed expression in (32). From (33) we have then $\lambda_{j}=\lim _{t \rightarrow+\infty} t^{-1} \log \left|y_{s}\left(t, \lambda_{j}, i\right)\right|$. Also from (33)

$$
s-1=\lim _{t \rightarrow+\infty} \frac{\log \left|y_{s}\left(t, \lambda_{j}, i\right) e^{-\lambda_{j} t}\right|}{\log t}
$$

which, in the terminology of Markus, is the type of $A(t)$ for the solution $y_{s}\left(t, \lambda_{j}, i\right)$. Finally the multiplicities of the characteristic exponents $\lambda_{j}$ as defined here in Definition 4 agree with those of Markus while the number of values of $i=1,2, \cdots, m_{j}$ such that $r_{i j} \geqq s$ is the multiplicity of the type $s-1$ corresponding to $\boldsymbol{\lambda}_{j}$.

Now suppose $A(t)$ is c.k. similar to a constant matrix; let $\lambda$ be a characteristic exponent and let $p(t)$ be one of the bounded functions $p_{1}(t, \lambda, i)$ such that inf $|p(t)|>0$. Then we have

$$
p^{\prime}(t)=A(t) p-\lambda p
$$

and also, since $\lambda$ is real, then

$$
p^{* \prime}(t)=p^{*}(t) A^{*}(t)-\lambda p^{*},
$$

where $D^{*}$ is the conjugate transpose of the matrix $D$. Let $u(t)=\left[p^{*}(t) p(t)\right]^{1 / 2}$ and we easily get from (35) and (36) that

$$
u u^{\prime}=p^{*} H p-\lambda u^{2},
$$

where $H(t)=\left[A(t)+A^{*}(t)\right] / 2$. Let $M(t)$ and $m(t)$ be the largest and smallest 
characteristic roots, respectively, of the Hermitian matrix $H(t)$ and we then have from (37)

$$
u u^{\prime} \leqq M(t) u^{2}-\lambda u^{2}
$$

and

$$
u u^{\prime} \geqq m(t) u^{2}-\lambda u^{2} .
$$

Integration of these inequalities, after division by $u^{2}$, leads to

$$
u_{0} \exp \int_{0}^{t}[m(s)-\lambda] d s \leqq u(t) \leqq u_{0} \exp \int_{0}^{t}[M(s)-\lambda] d s,
$$

for $t \geqq 0$, where $u_{0}=u(0)$. Now we may write

$$
\int_{0}^{t}[m(s)-\lambda] d s=t\left[t^{-1} \int_{0}^{t} m(s) d s-\lambda\right]
$$

and since $u(t)$ is bounded we conclude from (40) that

$$
\lim _{t \rightarrow+\infty} \sup \left[t^{-1} \int_{0}^{t} m(s) d s-\lambda\right] \leqq 0
$$

A similar analysis may be applied to the other exponential in (40), using the fact that inf $u(t)>0$, and we get finally

$$
\underset{t \rightarrow+\infty}{\operatorname{limsuv}} t^{-1} \int_{0}^{t} m(s) d s \leqq \lambda \leqq \liminf _{t \rightarrow+\infty} t^{-1} \int_{0}^{t} M(s) d s .
$$

This result is similar to Theorem 6 of Markus [4] except he assumes $A(t)$ is normal and he has lim sup for both bounds. We state our result formally as a

Corollary. Let $A(t) \in M_{n}$ be c.k. similar to a constant matrix. Let $M(t)$ and $m(t)$ be the maximum and minimum characteristic roots of $H(t)$ $=\left[A(t)+A^{*}(t)\right] / 2$. Then (41) holds for each characteristic exponent $\lambda$ for $A(t)$.

\section{REFERENCES}

1. E. A. Cu'-lington and N. Levinson, Theory of ordinary differential equations, New York, McGraw-Hill Book Company, Inc., 1955.

2. A. Liapounoff, Problème général de la stabilité du mouvement, reprinted by Princeton University Press as Annals of Mathematics Studies, no. 17.

3. J. C. Lillo, On almost periodic solutions of differential equations, Ann. of Math. vol. 69 (1959) pp. 467-485.

4. L. Markus, Continuous matrices and the stability of differential systems, Math. Z. vol. 62 (1955) pp. 310-319.

Iowa State University, AMES, IOWA 